\title{
UNIVERSALITY OF THE LOCAL SPACING DISTRIBUTION IN CERTAIN ENSEMBLES OF HERMITIAN WIGNER MATRICES
}

\author{
KURT JOHANSSON
}

\begin{abstract}
Consider an $N \times N$ hermitian random matrix with independent entries, not necessarily Gaussian, a so called Wigner matrix. It has been conjectured that the local spacing distribution, i.e. the distribution of the distance between nearest neighbour eigenvalues in some part of the spectrum is, in the limit as $N \rightarrow \infty$, the same as that of hermitian random matrices from GUE. We prove this conjecure for a certain subclass of hermitian Wigner matrices.
\end{abstract}

\section{INTRODUCTION AND MAIN RESULTS}

Consider a probability measure $\mathbb{P}_{N}$ on the space of all $N \times N$ hermitian matrices. We will be interested in the statistical properties of the spectrum as $N$ becomes large, in particular in features that are insensitive to the details of the particular sequence of probability measures we are considering. It is believed, on the basis of numerical simulations, that for many types of hermitian random matrix ensembles, i.e. choices of $\mathbb{P}_{N}$, the local statistical properties of the eigenvalues are the same as for the Gaussian Unitary Ensemble (GUE), where $d \mathbb{P}_{N}(M)=Z_{N}^{-1} \exp \left(-\frac{N}{2} \operatorname{Tr} M^{2}\right) d M$. Here $d M$ is Lebesgue measure on the space $\mathcal{H}_{N} \sim \mathbb{R}^{N^{2}}$ of all $N \times N$ hermitian matrices. The asymptotic eigenvalue density as $N \rightarrow \infty$ (density of states) is given by the Wigner semicircle law $\rho(t)=\frac{1}{2 \pi} \sqrt{\left(4-t^{2}\right)_{+}}$. Let $\rho_{N}\left(x_{1}, \ldots, x_{N}\right)$ be the induced probability density on the eigenvalues. The semicircle law is the limit of the one-dimensional marginal density as $N \rightarrow \infty$. The $m$ - point correlation function

$$
R_{m}^{(N)}\left(x_{1}, \ldots, x_{m}\right)=\frac{N !}{(N-m) !} \int_{\mathbb{R}^{N-m}} \rho_{N}(x) d x_{m+1} \ldots d x_{N},
$$

is given by, 20] ch. 5, 29],

$$
R_{m}^{(N)}\left(x_{1}, \ldots, x_{m}\right)=\operatorname{det}\left(K_{N}\left(x_{i}, x_{j}\right)\right)_{i, j=1}^{m},
$$

where the kernel $K_{N}(x, y)$ is given by

$$
K_{N}(x, y)=\frac{\kappa_{N-1}}{\kappa_{N}} \frac{p_{N}(x) p_{N-1}(y)-p_{N-1}(x) p_{N}(y)}{x-y} e^{-N\left(x^{2}+y^{2}\right) / 4} .
$$

Here $p_{N}(x)=\kappa_{N} x^{N}+\ldots$ are the normalized orthogonal polynomials with respect to the weight function $\exp \left(-N x^{2} / 2\right)$ on $\mathbb{R}$ (rescaled Hermite polynomials). From these formulas, and Plancherel-Rotach asymptotics for the Hermite polynomials it follows that

$$
\lim _{N \rightarrow \infty} \frac{1}{(N \rho(u))^{m}} R_{m}^{(N)}\left(u+\frac{t_{1}}{N \rho(u)}, \ldots, u+\frac{t_{m}}{N \rho(u)}\right)=\operatorname{det}\left(\frac{\sin \pi\left(t_{i}-t_{j}\right)}{\pi\left(t_{i}-t_{j}\right)}\right)_{i, j=1}^{m}
$$


if $\rho(u)>0$. It has been proved, [22], [7], [3], that this is also true in other invariant ensembles of the form $d \mathbb{P}_{N}(M)=Z_{N}^{-} \exp (-N \operatorname{Tr} V(M)) d M$. The orthogonal polynomials in (1.3) are then replaced by polynomials orthogonal with respect to $\exp (-N V(x))$ on $\mathbb{R}$. That the ensemble is invariant means that the probability measure is invariant under the conjugation $M \rightarrow U^{-1} M U$, with a unitary matrix $U$. Sufficient control of the limit (1.4) for all $m \geq 1$, makes it possible to determine the asymptotic spacing distribution, i.e. distances between nearest neighbour eigenvalues, see [7]. More precisely, let $\left\{t_{N}\right\}$ be a sequence such that $t_{N} \rightarrow \infty$ but $t_{N} / N \rightarrow 0$ as $N \rightarrow \infty$ and define, [17], []], $S_{N}(s, x), s \geq 0, x \in \mathbb{R}^{N}$, to be the symmetric function, which for $x_{1}<\cdots<x_{N}$ is defined by

$$
S_{N}(s, x)=\frac{1}{2 t_{N}} \#\left\{1 \leq j \leq N-1 ; x_{j+1}-x_{j} \leq \frac{s}{N \rho(u)},\left|x_{j}-u\right| \leq \frac{t_{N}}{N \rho(u)}\right\} .
$$

Given an hermitian matrix $M$ let $x_{1}(M)<\ldots x_{N}(M)$ be its eigenvalues; we write $x(M)=\left(x_{1}(M), \ldots, x_{N}(M)\right)$. Then it is proved in 7 that

$$
\lim _{N \rightarrow \infty} \mathbb{E}_{N}\left[S_{N}(s, x(M))\right]=\int_{0}^{s} p(\sigma) d \sigma
$$

for a large class of invariant ensembles. Here $p(\sigma)$ is the density of the $\beta=2$ local spacing distribution, the Gaudin distribution, given by the probability density

$$
p(s)=\frac{d^{2}}{d s^{2}} \operatorname{det}(I-K)_{L^{2}(0, s)},
$$

where $K$ is the operator on $L^{2}(0, s)$ with kernel $K(t, s)=\sin \pi(t-s) / \pi(t-s)$, the sine kernel, see [20].

The aim of the present paper is to extend (1.4) and (1.6) to other, non-invariant ensembles. It is conjectured, see 20] p.9, that (1.4) and (1.6) should hold also for so called Wigner matrices where the elements are independent but not necessarily Gaussian variables. In this case the probability measure is not invariant under conjugation by unitary matrices. For other results on Wigner matrices see for example [2], [18], [19], [25, [27 and [28]. In particular, in [26] the universality of the fluctuations of the largest eigenvalue is established. To be more precise, consider the complex random variables $w_{j k}, 1 \leq j \leq k$ with independent laws $P_{j k}=P_{j k}^{R} \otimes P_{j k}^{I}$, where $P_{j j}^{I}=\delta_{0}$. Let $\mathcal{W}^{p}$, a class of Wigner ensembles, denote the class of all $\left\{P_{j k}\right\}_{1 \leq j \leq k}$ which satisfy

$$
\int z d P_{j k}(z)=0 \quad, \quad \int|z|^{2} d P_{j k}(z)=\sigma^{2}
$$

for all $1 \leq j \leq k$, and furthermore

$$
\sup _{j, k} \int|z|^{p} d p_{j k}(z)<\infty
$$

If $w_{k j}=\bar{w}_{j k}, W=\left(w_{j k}\right)_{j, k=1}^{N}$ is an $N \times N$ hermitian Wigner matrix .

Fix $a>0$ and let $\phi_{a}(t)=\left(\pi a^{2}\right)^{-1 / 2} \exp \left(-t^{2} / a^{2}\right)$ be a Gaussian density function. Define $Q_{j k}^{R, I}=\phi_{a} * P_{j k}^{R, I}, 1 \leq j<k, Q_{j j}^{R}=\phi_{a \sqrt{2}} * P_{j j}^{R}, j \geq 1$ and $Q_{j j}^{I}=\delta_{0}$. Then $Q$ is also a Wigner ensemble and we let $\mathcal{W}_{a}^{p}$ denote the subclass of $\mathcal{W}^{p}$ obtained in this way. Note that although $\mathcal{W}_{a}^{p}$ does not contain all Wigner ensembles it does contain cases where the distribution of the matrix elements have very different shapes, so in this sense it is rather broad, and proving universality in $\mathcal{W}_{a}^{p}$ clearly shows that the universality is not restricted to the invariant ensembles. Another way to describe 
this ensemble of random matrices is as follows. Let $V$ be a GUE-matrix with the probability measure $Z_{N}^{-1} \exp \left(-\frac{1}{2} \operatorname{Tr} V^{2}\right) d V$ and let $W$ be an $N \times N$ Wigner matrix with distribution $P \in \mathcal{W}^{p}$, i.e. the law of $w_{j k}$ is $P_{j k}$. We will assume that the variance $\sigma^{2}=1 / 4$, which can always be achieved by rescaling. Then $W+a V$ has the distribution $Q$, and we write

$$
M=\frac{1}{\sqrt{N}}(W+a V) .
$$

We can think of this in terms of Dyson's Brownian motion model, [8], $W+a V$ is obtained from $W$ by letting the matrix elements execute a Brownian motion for a time $a^{2}$, see sect. 2. If $P \in \mathcal{W}^{p}$ and $W$ is is an $N \times N$ hermitian matrix we let $P^{(N)}$ denote the distribution of $H=W / \sqrt{N}=\left(h_{j k}\right)$, i.e.

$$
d P^{(N)}(H)=\prod_{1 \leq j \leq k \leq N} d P_{j k}\left(\sqrt{N} h_{j k}\right) .
$$

The matrix $M$ has the distribution $Q^{(N)}$, which is given by

$$
d Q^{(N)}(M)=2^{-N / 2}\left(\frac{N}{\pi a^{2}}\right)^{N^{2} / 2}\left(\int_{\mathcal{H}_{N}} e^{-\frac{N}{2 a^{2}} \operatorname{Tr}(M-H)^{2}} d P^{(N)}(H)\right) d M,
$$

and this is the measure we will study. The asymptotic distribution of the eigenvalues $x_{1}, \ldots, x_{N}$ of $M$ is the semicircle law

$$
\rho(u)=\frac{2}{\pi\left(1+4 a^{2}\right)} \sqrt{\left(1+4 a^{2}-u^{2}\right)_{+}} .
$$

The following proposition will be proved in sect. 2 using an argument from [4], [5].

Proposition 1.1. The symmetrized eigenvalue measure on $\mathbb{R}^{N}$ induced by $Q^{(N)}$ has a density

$$
\rho_{N}(x)=\int_{\mathcal{H}_{N}} \rho_{N}(x ; y(H)) d P^{(N)}(H)
$$

where

$$
\rho_{N}(x ; y)=\left(\frac{N}{2 \pi a^{2}}\right)^{N / 2} \frac{\Delta_{N}(x)}{\Delta_{N}(y)} \operatorname{det}\left(e^{-\frac{N}{2 a^{2}}\left(x_{j}-y_{k}\right)^{2}}\right)_{j, k=1}^{N}
$$

and $\Delta_{N}(x)=\prod_{1 \leq i<j \leq N}\left(x_{i}-x_{j}\right)$ is the Vandermonde determinant.

The main result of the present paper is that for Wigner ensembles from $\mathcal{W}_{a}^{p}$ we can prove (1.4) and (1.6), and thus extend the universality to a rather broad class of Wigner matrices.

Theorem 1.2. Fix $a>0$ and assume that $|u| \leq \sqrt{1 / 2+2 a^{2}}$. Let $R_{M}^{(N)}\left(x_{1}, \ldots, x_{m}\right)$ be the correlation functions, defined by (1.1), of the eigenvalue measure $\rho_{N}$, 1.12), for $Q^{(N)}$, 1.10). Let $f \in L_{c}^{\infty}\left(\mathbb{R}^{m}\right)$, the set of all $L^{\infty}$ functions on $\mathbb{R}^{m}$ with compact support, and set for $x \in \mathbb{R}^{N}$

$$
(S f)(x)=\sum_{i_{1}, \ldots, i_{m}}^{\prime} f\left(x_{i_{1}}, \ldots, x_{i_{m}}\right)
$$


where the sum is over all distinct indices from $\{1, \ldots, N\}$. If $Q \in \mathcal{W}_{a}^{p}$ with $p>$ $2(m+2)$, then

$$
\begin{aligned}
& \lim _{N \rightarrow \infty} \int_{\mathcal{H}_{N}}(S f)\left(N \rho(u)\left(x_{1}(M)-u\right), \ldots N \rho(u)\left(x_{N}(M)-u\right)\right) d Q^{(N)}(M) \\
& \lim _{N \rightarrow \infty} \int_{\mathbb{R}^{m}} f\left(t_{1}, \ldots, t_{m}\right) \frac{1}{(N \rho(u))^{m}} R_{m}^{(N)}\left(u+\frac{t_{1}}{N \rho(u)}, \ldots, u+\frac{t_{m}}{N \rho(u)}\right) d^{m} t \\
& =\int_{\mathbb{R}^{m}} f\left(t_{1}, \ldots, t_{m}\right) \operatorname{det}\left(\frac{\sin \pi\left(t_{i}-t_{j}\right)}{\pi\left(t_{i}-t_{j}\right)}\right)_{i, j=1}^{m} d^{m} t .
\end{aligned}
$$

The condition on $u$ is made just to simplify the saddle-point argument in sect. 3 ; the result should hold for any $u$ with $\rho(u)>0$.

We can also prove that the spacing distribution is the same as for GUE.

Theorem 1.3. Fix any $a>0$ and assume that $Q \in \mathcal{W}_{a}^{6+\epsilon}, \epsilon>0$. Let $S_{N}(s, x)$ be defined by (1.5). Then, for any $s \geq 0$,

$$
\lim _{N \rightarrow \infty} \int_{\mathcal{H}_{N}} S_{N}(s, x(M)) d Q^{(N)}(M)=\int_{0}^{s} p(\sigma) d \sigma
$$

where $p(s)$ is given by (1.7).

The theorems will be proved in sect. 4 after the preparatory work in sect. 2 and 3.

\section{The CORRELATION FUnCTIONS}

We will start by proving Proposition 1.1 using the Harish-Chandra/ItzyksonZuber formula following [4], [5]. After that we will give a formula for the correlation functions of $\rho_{N}(x ; y)$, which is very close to the formula in [6], but our derivation will be different. A central role will be played by non-intersecting one-dimensional Brownian motions and we will use the formulas of Karlin and McGregor. Also we will discuss the relation to Dyson's Brownian motion model. This connection can be found in [9] and we will only give an outline.

Proof. Let $F(x)$ be a continuous symmetric function on $\mathbb{R}^{N}$. By Fubini's theorem

$$
\int_{\mathcal{H}_{N}} F(x(M)) d Q^{(N)}(M)=c_{N}^{(1)} \int_{\mathcal{H}_{N}}\left(\int_{\mathcal{H}_{N}} F(x(M)) e^{-\frac{N}{2 a^{2}} \operatorname{Tr}(M-H)^{2}} d M\right) d P^{(N)}(H)
$$

with $c_{N}^{(1)}=2^{-N / 2}\left(N / \pi a^{2}\right)^{N^{2} / 2}$. In the right hand side of $(2.1)$ we make the substitution $M=U^{-1} R U$, with $U \in U(N)$ and $R \in \mathcal{H}_{N}$, and then integrate over $U(N)$. If we use Fubini's theorem again, we obtain

$$
c_{N}^{(1)} \int_{\mathcal{H}_{N}}\left(\int_{\mathcal{H}_{N}} F(x(R))\left(\int_{U(N)} e^{-\frac{N}{2 a^{2}} \operatorname{Tr}\left(U^{-1} R U-H\right)^{2}} d U\right) d R\right) d P^{(N)}(H) .
$$

Here we have also used the fact that $d M=d R$. The integral over $U(N)$ can now be evaluated using the Harish-Chandra/Itzykson-Zuber formula, [1], 13], see also 20 A.5. We obtain the integral

$$
c_{N}^{(1)} c_{N}^{(2)} \int_{\mathcal{H}_{N}}\left(\int_{\mathcal{H}_{N}} F(x) \frac{1}{\Delta_{N}(x) \Delta_{N}(y)} \operatorname{det}\left(e^{-N\left(x_{j}-y_{k}\right)^{2} / 2 a^{2}}\right)_{j, k=1}^{N} d R\right) d P^{(N)}(H),
$$


where $y_{1}, \ldots, y_{N}$ are the eigenvalues of $H$ and $c_{N}^{(2)}=\left(a^{2} / N\right)^{N(N-1) / 2} \prod_{j=1}^{N} j$ !. The integrand in the middle integral depends only on the eigenvalues $x$ of $R$ and hence we can integrate out the other degrees of freedom in the standard way, [20] ch. 3, and obtain, after using Fubini's theorem,

$$
\begin{aligned}
& \int_{\mathcal{H}_{N}} F(x(M)) d Q^{(N)}(M) \\
& (2.2) \\
& =c_{N}^{(1)} c_{N}^{(2)} c_{N}^{(3)} \int_{\mathcal{H}_{N}}\left(\int_{\mathbb{R}^{N}} F(x) \frac{\Delta_{N}(x)}{\Delta_{N}(y)} \operatorname{det}\left(e^{-N\left(x_{j}-y_{k}\right)^{2} / 2 a^{2}}\right)_{j, k=1}^{N} d^{N} x\right) d P^{(N)}(H)
\end{aligned}
$$

with $c_{N}^{(3)}=\pi^{N(N-1) / 2} \prod_{j=1}^{N}(j !)^{-1}$. We see that $c_{N}^{(1)} c_{N}^{(2)} c_{N}^{(3)}=\left(N / 2 \pi a^{2}\right)^{N / 2}$ and since (2.2) holds for arbitrary bounded, continuous, symmetric $F(x)$ we have proved that the symmetrized eigenvalue measure is given by 1.12. This proves proposition 1.1 .

Let $p_{t}(x, y)$ be the transition probability of a Markov process $X(t)$ on $\mathbb{R}$ with continuous paths. Consider $N$ independent copies of the process $\left(X_{1}(t), \ldots, X_{N}(t)\right)$ and assume that this is a strong Markov process in $\mathbb{R}^{N}$. Suppose that the particles start at positions $y_{1}<\cdots<y_{N}$ at time 0 . The probability density that they are at positions $x_{1}<\cdots<x_{N}$ at time $S$ given that their paths have not intersected anytime during the time interval $[0, S]$ is, by a theorem of Karlin and McGregor, 16.

$$
\operatorname{det}\left(P_{S}\left(y_{j}, x_{k}\right)\right)_{j, k=1}^{N} .
$$

Hence, the conditional probability density that the particles are at positions $y_{1}<$ $\cdots<y_{N}$ at time 0 , at positions $x_{1}<\cdots<x_{N}$ at time $S$, at positions $z_{1}<\cdots<z_{N}$ at time $S+T$, given that their paths have not intersected in the time interval $[0, S+T]$ is

$$
q_{S, T}(x ; y ; z) \doteq \frac{1}{\mathcal{Z}_{N}} \operatorname{det}\left(P_{S}\left(y_{j}, x_{k}\right)\right)_{j, k=1}^{N} \operatorname{det}\left(P_{T}\left(x_{j}, z_{k}\right)\right)_{j, k=1}^{N},
$$

where

$$
\mathcal{Z}_{N}=\int_{x_{1}<\cdots<x_{N}} \operatorname{det}\left(P_{S}\left(y_{j}, x_{k}\right)\right)_{j, k=1}^{N} \operatorname{det}\left(P_{T}\left(x_{j}, z_{k}\right)\right)_{j, k=1}^{N} d^{N} x
$$

we assume that $\mathcal{Z}_{N}>0$. Note that the expression (2.3) is a symmetric function of $x_{1}, \ldots, x_{N}$, so we can regard it as a probability measure on $\mathbb{R}^{N}$. Our next lemma shows that we can obtain $\rho_{N}(x ; y)$ defined by (1.13) as a limit of the measure in (2.3).

Lemma 2.1. Let $z_{j}=j-1,1 \leq j \leq N$ and let $p_{t}(x, y)=(2 \pi t)^{-1 / 2} \exp ((x-$ $\left.y)^{2} / 2 t\right)$ be the transition probability for Brownian motion. Then, for any $x \in \mathbb{R}^{N}$ and $y_{1}<\ldots, y_{N}$,

$$
\lim _{T \rightarrow \infty} q_{S, T}(x ; y ; z)=\frac{1}{(2 \pi S)^{N / 2}} \frac{\Delta_{N}(x)}{\Delta_{N}(y)} \operatorname{det}\left(e^{-\left(x_{j}-y_{k}\right)^{2} / 2 S}\right)_{j, k=1}^{N} \doteq q_{S}(x ; y) .
$$

Note that $\rho_{N}(x ; y)=q_{a^{2} / N}(x ; y)$. 
Proof. Write

$$
\begin{aligned}
& \operatorname{det}\left(P_{S}\left(y_{j}, x_{k}\right)\right)_{j, k=1}^{N} \operatorname{det}\left(P_{T}\left(x_{j}, z_{k}\right)\right)_{j, k=1}^{N} \\
& =\frac{1}{(2 \pi)^{N}(T S)^{N / 2}} \operatorname{det}\left(e^{-\left(x_{j}-y_{k}\right)^{2} / 2 S}\right)_{j, k=1}^{N} \prod_{j=1}^{N} e^{-\frac{x_{j}^{2}+z_{j}^{2}}{2 T}} \operatorname{det}\left(e^{x_{j} z_{k} / 2 T}\right)_{j, k=1}^{N} .
\end{aligned}
$$

Note that $\mathcal{Z}_{N}$ is the conditional probability density of going from $y_{1}<\ldots, y_{N}$ to $z_{1}<\ldots, z_{N}$ without collosions, i.e.

$$
\begin{aligned}
\mathcal{Z}_{N} & =\operatorname{det}\left(p_{S+T}\left(y_{j}, z_{k}\right)\right)_{j, k=1}^{N} \\
& =\frac{1}{(2 \pi)^{N / 2}(S+T)^{N / 2}} \prod_{j=1}^{N} e^{-\frac{y_{j}^{2}+z_{j}^{2}}{2(S+T)}} \operatorname{det}\left(e^{y_{j} z_{k} / 2(S+T)}\right)_{j, k=1}^{N}
\end{aligned}
$$

Now, since $z_{j}=j-1$, we have two Vandermonde determinants in (2.5) and (2.6). If we evaluate these, take the quotient between $(2.5)$ and $(2.6)$ and then take the limit $T \rightarrow \infty$, we obtain the right hand side of (2.4).

Proposition 1.1 and lemma 2.1 establish a link between the eigenvalue distribution of $M=(W+a V) / \sqrt{N}$ and the non-intersecting Brownian paths. If we set $S=a^{2} / N$, then the right hand side of (2.4) and (1.13) are identical; $y_{1}<\cdots<y_{N}$ are the eigenvalues of $H=W / \sqrt{N}$. This relation can also be seen in another way, which we will now outline. Let $X(t)=\left(x_{j k}(t)\right)_{j, k=1}^{N}$ be an $N \times N$ Hermitian matrix, where $\operatorname{Re} x_{j k}(t), \operatorname{Im} x_{j k}(t), j \leq k$ are independent Brownian motions with variance $\left(1+\delta_{j k}\right) / 2$. Assume that $X(0)=H$ is distributed according to $P^{(N)}$. Then the distribution of $X\left(a^{2} / N\right)$ is the same as that of $M=(W+a V) / \sqrt{N}$. Following Dyson, [8], see also [23], it is possible to derive a stochastic differential equation for the eigenvalues $\lambda_{1}(t), \ldots, \lambda_{N}(t)$ of $X(t)$,

$$
d \lambda_{i}=d B_{i}+\sum_{k \neq i} \frac{1}{\lambda_{i}-\lambda_{k}} d t
$$

where $B_{i}$ are independent standard Brownian motions on $\mathbb{R}$, and with the intial conditions $\lambda_{i}(0)=y_{i}, 1 \leq i \leq N$. We can also consider the problem of nonintersecting Brownian motions in a different way than that of Karlin and McGregor. Namely, let $K=\left\{x \in \mathbb{R}^{N} ; x_{1}<\cdots<x_{N}\right\}$ and consider Brownian motion in $\mathbb{R}^{N}$ starting at $y \in K$ and conditioned to remain in $K$ forever. As proved in [9], see also [12], [24], if $\lambda_{i}$ are the components of the $N$-dimensional conditioned Brownian motion they satisfy the stochastic differential equation (2.7) with the same initial conditions. This gives another way to obtain (1.13) without using the HarishChandra, Itzykson/Zuber formula. Actually, we can turn the argument around and give a proof of this formula.

We turn now to the computation of the correlation functions of the right hand side of (2.4), but we start more generally with (2.3). This can be analyzed using the techniques of [29], compare the analysis of the Schur measure, [21], in [14], and see also [15]. For completeness, let us outline the result we need from [29]. Let $(\Omega, \mu)$ be a measure space. Assume that $\phi_{j}, \psi_{j} \in L^{2}(\Omega, \mu), 1 \leq j \leq N$, and $f \in L^{\infty}(\Omega, \mu)$. Set

$$
Z_{N}[f]=\frac{1}{N !} \int_{\Omega_{N}} \operatorname{det}\left(\phi_{j}\left(x_{k}\right)\right)_{j, k=1}^{N} \operatorname{det}\left(\psi_{j}\left(x_{k}\right)\right)_{j, k=1}^{N} \prod_{j=1}^{N} f\left(x_{j}\right) d \mu\left(x_{j}\right)
$$


and

$$
A=\left(\int_{\Omega} \phi_{j}(x) \psi_{j}(x) d \mu(x)\right)_{j, k=1}^{N} .
$$

Proposition 2.2. (29]) Assume that $Z_{N}[1] \neq 0$. Then $A$ is invertible and we can define

$$
K_{N}(t, s)=\sum_{j, k=1}^{N} \psi_{k}(t)\left(A^{-1}\right)_{k j} \phi_{j}(s) .
$$

Then, for any $g \in L^{\infty}(\Omega, \mu)$,

$$
\frac{Z_{N}[1+g]}{Z_{N}[1]}=\operatorname{det}\left(I+K_{N} g\right)_{L^{2}(\Omega)}
$$

If we define a density on $\Omega^{N}$ by

$$
u_{N}(x)=\frac{1}{N ! Z_{N}[1]} \operatorname{det}\left(\phi_{j}\left(x_{k}\right)\right)_{j, k=1}^{N} \operatorname{det}\left(\psi_{j}\left(x_{k}\right)\right)_{j, k=1}^{N},
$$

then it has the correlation functions

$$
\frac{N !}{(N-M) !} \int_{\Omega^{N-m}} u_{N}(x) d x_{m+1} \ldots d x_{N}=\operatorname{det}\left(K_{N}\left(x_{i}, x_{j}\right)\right)_{i, j=1}^{N} .
$$

Proof. We will indicate the main steps in the proof of (2.9) of which (2.11) is a consequence, see [29]. Set

$$
B=\left(\int_{\Omega} \phi_{j}(x) \psi_{j}(x) g(x) d \mu(x)\right)_{j, k=1}^{N} .
$$

Then, by the formula

$$
Z_{N}[f]=\operatorname{det}\left(\int_{\Omega} \phi_{j}(x) \psi_{j}(x) f(x) d \mu(x)\right)_{j, k=1}^{N},
$$

which goes all the way back to [1, and which is not difficult to prove by expanding the determinants, we see that $\operatorname{det} A=Z_{N}[1] \neq 0$, so $A$ is invertible and

$$
\frac{Z_{N}[1+g]}{Z_{N}[1]}=\frac{\operatorname{det}(A+B)}{\operatorname{det} A}=\operatorname{det}\left(I+A^{-1} B\right) .
$$

Now,

$$
\left(A^{-1} B\right)_{j k}=\int_{\Omega} \psi_{k}(x)\left(\sum_{\ell=1}^{N}\left(A^{-1}\right)_{j \ell} \phi_{\ell}(x) g(x)\right) d \mu(x),
$$

and we define $T: \mathbb{C}^{N} \rightarrow L^{2}(\Omega, d \mu)$ and $S: L^{2}(\Omega, d \mu) \rightarrow \mathbb{C}^{N}$ by the kernels $T(x, k)=\psi_{k}(x)$ and $S(j, x)=\sum_{\ell=1}^{N}\left(A^{-1}\right)_{j \ell} \phi_{\ell}\left(x_{g}(x)\right.$. Then, by 2.12) and a determinant identity,

$$
\begin{aligned}
\frac{Z_{N}[1+g]}{Z_{N}[1]} & =\operatorname{det}(I+S T)_{\mathbb{C}^{N}}=\operatorname{det}(I+T S)_{L^{2}(\Omega, d \mu)} \\
& =\operatorname{det}\left(I+K_{N} g\right)_{L^{2}(\Omega, d \mu)},
\end{aligned}
$$

with $K_{N}$ given by (2.8). Note that $K_{N} g$, which means first multiplication by $g$ and then application of the operator on $L^{2}(\Omega, d \mu)$ with kernel $K_{N}$, is a finite rank operator. 
Observe now that if we take $\Omega=\mathbb{R}, d \mu(x)=d x, \phi_{j}(x)=P_{T}\left(x, z_{j}\right)$ and $\psi_{j}(x)=$ $p_{S}\left(y_{j}, x\right)$, then $(2.3)$ is a probability density of the form (2.10) and we can apply the proposition. Note that

$$
(A)_{j k}=\int_{\mathbb{R}} p_{T}\left(x, z_{j}\right) p_{S}\left(y_{k}, x\right) d x=p_{S+T}\left(y_{k}, z_{j}\right)
$$

The kernel which gives the correlation functions is

$$
K_{N}^{S, T}(u, v)=\sum_{k=1}^{N} p_{S}\left(y_{k}, v\right)\left(\sum_{j=1}^{N}\left(A^{-1}\right)_{j k} p_{T}\left(u, z_{j}\right)\right) .
$$

Let $A_{k}(v)$ be the matrix we obtain from $A$ by replacing column $k$ by $\left(p_{T}\left(v, z_{1}\right) \ldots p_{T}\left(v, z_{N}\right)\right)^{T}$. Then, by Kramers' rule,

$$
K_{N}^{S, T}(u, v)=\sum_{k=1}^{N} p_{S}\left(y_{k}, v\right) \frac{\operatorname{det} A_{k}(v)}{\operatorname{det} A} .
$$

This formula and proposition 2.2 is the basis for the next proposition. The result is closely related to the result derived in [6] by different methods.

Proposition 2.3. The correlation functions for $q_{S}(x ; y)$ defined by (2.4) are given by

$$
\begin{aligned}
R_{m}^{N}\left(x_{1}, \ldots, x_{m} ; y\right) & \doteq \frac{N !}{(N-m) !} \int_{\mathbb{R}^{N-m}} q_{S}(x ; y) d x_{m+1} \ldots d x_{N} \\
& =\operatorname{det}\left(K_{N}^{S}\left(x_{i}, x_{j} ; y\right)\right)_{i, j=1}^{m},
\end{aligned}
$$

where

$$
\begin{aligned}
& K_{N}^{S}(u, v ; y)=\frac{e^{\left(v^{2}-u^{2}\right) / 2 S}}{(v-u) S(2 \pi i)^{2}} \int_{\gamma} d z \int_{\Gamma} d w\left(1-e^{(v-u) z / S}\right) \\
& \times \frac{1}{z}\left(w+z-v-S \sum_{j} \frac{y_{j}}{\left(w-y_{j}\right)\left(z-y_{j}\right)}\right) e^{\left(w^{2}-2 v w-z^{2}+2 u z\right) / 2 S} .
\end{aligned}
$$

Here $\gamma$ is the union of the curves $t \rightarrow-t+i \omega, t \in \mathbb{R}$ and $t \rightarrow t-i \omega, t \in \mathbb{R}$ with a fixed $\omega>0$, and $\Gamma: \mathbb{R} \ni t \rightarrow i t$.

Proof. We have to show that with $p_{t}(u, v)=(2 \pi t)^{-1 / 2} \exp \left(-(u-v)^{2} / 2 t\right)$ and $z_{j}=j-1$ the limit of the right hand side of (2.13) as $T \rightarrow \infty$ can be written as (2.15). The result then follows from lemma 2.1, proposition 2.2 and the dominated convergence theorem. We see that

$$
\operatorname{det} A=\frac{1}{(2 \pi(S+T))^{N / 2}} \prod_{j=1}^{N} e^{-\frac{z_{j}^{2}+y_{j}^{2}}{2(S+T)}} \prod_{1 \leq i<j \leq N}\left(e^{\frac{y_{j}}{S+T}}-e^{\frac{y_{i}}{S+T}}\right)
$$

by the formula for a Vandermonde determinant. Let $\Gamma_{M}^{*}$ be the curve $t \rightarrow t+i M$, $t \in \mathbb{R}, M$ fixed. Then

$$
p_{T}\left(z_{j}, v\right)=\frac{1}{\sqrt{2 \pi T}} e^{-\frac{z_{j}^{2}}{2(S+T)}-\frac{v^{2}}{2 T}} \frac{1}{\sqrt{2 \pi}} \int_{\Gamma_{M}^{*}} e^{-\frac{\tau^{2}}{2}+z_{j}\left(\frac{v}{T}+i \tau \sqrt{\left.\frac{S}{2 T(S+T)}\right)}\right.} d \tau
$$


Hence,

$$
\begin{aligned}
\operatorname{det} A_{k}(v) & =\frac{1}{2 \pi \sqrt{T}} \frac{1}{(2 \pi(S+T))^{(N-1) / 2}}\left(\prod_{j=1}^{N} e^{-\frac{z_{j}^{2}}{2(S+T)}}\right)\left(\prod_{j \neq k} e^{-\frac{y_{j}^{2}}{2(S+T)}}\right) \\
& \times e^{-\frac{v^{2}}{2 T}} \int_{\Gamma_{M}^{*}} e^{-\frac{\tau^{2}}{2}} \operatorname{det} \tilde{A}_{k}(v) d \tau
\end{aligned}
$$

where $\tilde{A}_{k}(v)$ is the matrix we get from $\left(\exp \left(\frac{z_{j} y_{k}}{S+T}\right)\right)_{j, k=1}^{N}$ by replacing column $k$ by $\left(\exp \left(z_{j}\left(\frac{v}{T}+i \tau \sqrt{\frac{S}{2 T(S+T)}}\right)\right)\right)_{j=1}^{N}$. Since $z_{j}=j-1$ we have a Vandermonde determinant and we obtain

$$
\begin{aligned}
\operatorname{det} A_{k}(v) & =\sqrt{\frac{S+T}{T}} \frac{1}{(2 \pi(S+T))^{N / 2}}\left(\prod_{j=1}^{N} e^{-\frac{z_{j}^{2}}{2(S+T)}}\right)\left(\prod_{j \neq k} e^{-\frac{y_{j}^{2}}{2(S+T)}}\right) \\
& \times e^{-\frac{v^{2}}{2 T}} \frac{1}{\sqrt{21}} \int_{\Gamma_{M}^{*}} e^{-\frac{\tau^{2}}{2}} \prod_{1 \leq i<j \leq N}\left(e^{\frac{y_{j}}{S+T}}-e^{\frac{y_{i}}{S+T}}\right) d \tau,
\end{aligned}
$$

where $y_{k}$ should be replaced by $(S+T)\left(\frac{v}{T}+i \tau \sqrt{\frac{S}{2 T(S+T)}}\right)$. Take the quotient of (2.16) and (2.17) and let $T \rightarrow \infty$. This gives

$$
\lim _{T \rightarrow \infty} \frac{\operatorname{det} A_{k}(v)}{\operatorname{det} A}=\frac{1}{\sqrt{2 \pi}} \int_{\Gamma_{M}^{*}} e^{-\frac{\tau^{2}}{2}} \prod_{j \neq k}\left(\frac{v+i \sqrt{S} \tau-y_{j}}{y_{k}-y_{j}}\right) d \tau .
$$

Choose $M$ so that $v-\sqrt{S} M=L$, where $L$ is given, and make the change of variables $w=v+i \sqrt{S} \tau$. Then

$$
\lim _{T \rightarrow \infty} \frac{\operatorname{det} A_{k}(v)}{\operatorname{det} A}=\frac{1}{i \sqrt{2 \pi S}} \int_{\Gamma_{L}} e^{\frac{(w-v)^{2}}{2 S}} \prod_{j \neq k}\left(\frac{w-y_{j}}{y_{k}-y_{j}}\right) d w
$$

where $\Gamma_{L}: t \rightarrow L+i t, t \in \mathbb{R}$. Thus, using (2.13),

$$
K_{N}^{S}(u, v ; y)=\frac{1}{2 \pi i S} \sum_{k=1}^{N} e^{-\left(y_{k}-u\right)^{2} / 2 S} \int_{\Gamma_{L}} e^{\frac{(w-v)^{2}}{2 S}} \prod_{j \neq k}\left(\frac{w-y_{j}}{y_{k}-y_{j}}\right) d w .
$$

Let $\gamma$ be a curve surrounding $y_{1}, \ldots, y_{N}$ and choose $L$ so large that $\gamma$ and $\Gamma$ do not intersect. The residue theorem gives

$$
\frac{1}{2 \pi i} \int_{\gamma} \frac{e^{-(z-u)^{2} / 2 S}}{w-z} \prod_{j=1}^{N} \frac{w-y_{j}}{z-y_{j}} d z=\sum_{k=1}^{N} e^{-\left(y_{k}-u\right)^{2} / 2 S} \prod_{j \neq k}\left(\frac{w-y_{j}}{y_{k}-y_{j}}\right)
$$

for all $w \in \Gamma_{L}$. Thus,

$$
K_{N}^{S}(u, v ; y)=\frac{e^{\frac{v^{2}-u^{2}}{2 S}}}{(2 \pi i)^{2} S} \int_{\gamma} d z \int_{\Gamma_{L}} d w e^{\frac{1}{2 S}\left(w^{2}-2 v w-z^{2}+2 u z\right)} \frac{1}{w-z} \prod_{j=1}^{N} \frac{w-y_{j}}{z-y_{j}}
$$

In (2.18) we make the change of variables $z \rightarrow b z, w \rightarrow b w$ with $b \in \mathbb{R}$ close to 1 . This will modify the contours but we can use Cauchy's theorem to deform back to 
$\gamma$ and $\Gamma_{L}$. Now, take the derivative with respect to $b$ and then put $b=1$. This gives the equation

$$
\begin{aligned}
0 & =K_{N}^{S}(u, v ; y)+\frac{e^{\frac{v^{2}-u^{2}}{2 S}}}{(2 \pi i)^{2} S^{2}} \int_{\gamma} d z \int_{\Gamma_{L}} d w \frac{1}{w-z} e^{\frac{1}{2 S}\left(w^{2}-2 v w-z^{2}+2 u z\right)} \\
& \times\left[w^{2}-z^{2}+u z-v w+S \sum_{j=1}^{N}\left(\frac{w}{w-y_{j}}-\frac{z}{z-y_{j}}\right)\right] \prod_{j=1}^{N} \frac{w-y_{j}}{z-y_{j}} .
\end{aligned}
$$

This can be written

$$
\begin{aligned}
& \frac{\partial}{\partial u}\left((u-v) K_{N}^{S}(u, v ; y)\right)=-\frac{e^{\frac{v^{2}-u^{2}}{2 S}}}{(2 \pi i)^{2} S^{2}} \int_{\gamma} d z \int_{\Gamma_{L}} d w \\
& {\left[w+z-v-S \sum_{j=1}^{N} \frac{y_{j}}{\left(w-y_{j}\right)\left(z-y_{j}\right)}\right] e^{\left(w^{2}-2 v w-z^{2}\right) / 2 S} e^{u z / S} \prod_{j=1}^{N} \frac{w-y_{j}}{z-y_{j}},}
\end{aligned}
$$

and integration of this formula gives (2.15). In this last formula we can choose $L$ arbitrarily and take $\gamma$ to be the curve in the proposition by using Cauchy's formula, This completes the proof.

We now take $S=a^{2} / N$ and set

$$
\mathcal{K}_{N}(u, v ; y)=e^{\frac{N\left(u^{2}-v^{2}\right)}{2 a^{2}}+\omega(u-v)} K_{N}^{a^{2} / N}(u, v ; y),
$$

where $\omega$ is a constant that will be specified later. Note that we can replace $K_{N}^{a^{2} / N}$ with $\mathcal{K}_{N}$ in (2.14) without changing the correlation functions, so we can just as well work with $\mathcal{K}_{N}$. Set

$$
\begin{aligned}
& f_{N}(z)=\frac{1}{2 a^{2}}\left(z^{2}-2 u z\right)+\frac{1}{N} \sum_{j=1}^{N} \log \left(z-y_{j}\right) \\
& g_{N}(z, w)=\frac{1}{a^{2} z}\left(w+z-u-\frac{a^{2}}{N} \sum_{j=1}^{N} \frac{y_{j}}{\left(w-y_{j}\right)\left(z-y_{j}\right)}\right) \\
& h(z, w)=\frac{e^{\omega(u-v)}}{N \rho(u)(v-u)} e^{\frac{N}{a^{2}}(u-v) w}\left(e^{N(u-v) w / a^{2}}-e^{N(u-v)(w-z) / a^{2}}\right),
\end{aligned}
$$

so that

$$
\mathcal{K}_{N}(u, v ; y)=N \rho(u) \int_{\gamma} \frac{d z}{2 \pi i} \int_{\Gamma} \frac{d w}{2 \pi i} h(z, w) g_{N}(z, w) e^{N\left(f_{N}(w)-f_{N}(z)\right)} .
$$

These are the formulas we will use in the asymptotic analysis. A straightforward computation shows that

$$
g_{N}(z, w)=\frac{1}{z} f_{N}^{\prime}(z)+\frac{f_{N}^{\prime}(z)-f_{N}^{\prime}(w)}{z-w} .
$$

\section{Asymptotics} law

The eigenvalues $y_{1}, \ldots, y_{N}$ of the Wigner matrix $H$ converge to the semicircle

$$
\sigma(t)=\frac{2}{\pi} \sqrt{1-t^{2}}, \quad|t| \leq 1
$$


In order to be able to perform the saddle point analysis of (2.20) we need uniform control of the convergence of $f_{N}(z)$ to its limit

$$
f(z)=\frac{1}{2 a^{2}}\left(z^{2}-2 u z\right)+\int_{-1}^{1} \log (z-t) \sigma(t) d t .
$$

In order to show this we must start with some probability estimates. Write $\Omega_{R, \eta}=$ $\{z \in \mathbb{C} ;|\operatorname{Re} z| \leq R, \eta \leq|\operatorname{Im} z| \leq R\}$.

Lemma 3.1. Let $F \in L^{\infty}\left(\mathbb{R}^{N}\right)$ be symmetric and let $\eta>0$ and $R>0$ be given. Assume that $P \in \mathcal{W}^{p}, p>4$ and $0<\xi<\min \left(\frac{1}{2}-\frac{2}{p}, \frac{1}{16}\right)$. Then, there is a probability measure $\tilde{P}^{(N)}$ on $\mathcal{H}_{N}$ such that

$$
\left|\int_{\mathcal{H}_{N}} F(x(H)) d P^{(N)}(H)-\int_{\mathcal{H}_{N}} F(x(H)) d \tilde{P}^{(N)}(H)\right| \leq N^{2-p\left(\frac{1}{2}-\xi\right)}\|F\|_{\infty},
$$

and

$$
\sup _{z \in \Omega_{R, \eta}}\left|\frac{1}{N} \operatorname{Tr} \log (z-H)-\int_{-1}^{1} \log (z-t) \sigma(t) d t\right| \leq C N^{-\xi}
$$

a.s. with respect to $\tilde{P}^{(N)}$.

Proof. Given $P \in \mathcal{W}^{p}$ we introduce a cut-off $L>0$ and define a new probability measure $P_{L} \in \mathcal{W}^{p}$ by

$$
d P_{L, j k}^{R, I}(t)=\frac{1}{d_{L, j k}} \chi_{[-L, L]}(t) d P_{j k}^{R, I}(t) \quad, 1 \leq j \leq k
$$

where $d_{L, j k}$ is a normalization constant. Note that $P_{L, j k}$ is supported in $K=$ $[-L, L]^{2}$. Set $d_{L}^{(N)}=\prod_{1 \leq j \leq k \leq N} d_{L, j k}$. Then,

$$
\begin{aligned}
\left|\int_{\mathcal{H}_{N}} F(x(H)) d P^{(N)}(H)-\int_{\mathcal{H}_{N}} F(x(H)) d P_{L}^{(N)}(H)\right| & \leq\|F\|_{\infty}\left(1-d_{L}^{(N)}\right)\left(1+\frac{1}{d_{L}^{(N)}}\right) \\
& \leq \frac{C N^{2}}{L^{p}}\|F\|_{\infty}
\end{aligned}
$$

for some constant $C$. The last estimate follows from

$$
1-d_{L}^{(N)}=P\left[\text { some }\left|W_{j k}\right| \geq L\right] \leq N^{2} \sup _{1 \leq j \leq k} \frac{E\left[\left|W_{j k}\right|^{p}\right]}{L^{p}} \leq \frac{C N^{2}}{L^{p}}
$$

by (1.9). Set $D_{N}=\Omega_{R, \eta} \cap \frac{1}{N} \mathbb{Z}^{2}$ and note that $\# D_{N} \leq C N^{2}$ for some constant $C$ that only depend on $R, \eta$. For a given function $f$ set

$$
A_{N}(f ; \delta)=\left\{H \in \mathcal{H}_{N} ;\left|\frac{1}{N} \operatorname{Tr}(f(H))-\int_{-1}^{1} f(t) d \sigma(t)\right| \leq \delta\right\},
$$

where $\sigma(t)$ is the semicircle law (3.5). Set

$$
A_{N}(\delta)=\bigcap_{z \in D_{N}} A_{N}\left(f_{z}, \delta\right)
$$

where $f_{z}(t)=\log (z-t)$ (principal branch). To estimate the probability of $A_{N}(\delta)$ under $P^{(N)}$ we will use a result of Guionnet and Zeitouni, [10]. Let

$$
|f|_{\mathcal{L}}=\sup _{t, s \in \mathbb{R}} \frac{|f(x)-f(y)|}{|x-y|}
$$


and $\|f\|_{\mathcal{L}}=\|f\|_{\infty}+|f|_{\mathcal{L}}$. Then, by [10], corollary 1.6a), and the discussion before this corollary, given $\epsilon>0$, there are positive constants $C_{0}(\epsilon), C_{1}$ and $C_{2}$ such that if we write

$$
\delta_{1}(N)=C_{1} L^{2}|f|_{\mathcal{L}} N^{-1}+C_{2}(\epsilon)\|f\|_{\mathcal{L}} N^{-1 / 4+\epsilon},
$$

then

$$
P_{L}^{(N)}\left[\left|\frac{1}{N} \operatorname{Tr} f(H)-\int_{-1}^{1} f(t) \sigma(t) d t\right| \geq \delta\right] \leq 4 \exp \left[-\frac{C_{2} N^{2}}{L^{4}|f|_{\mathcal{L}}}\left(\delta-\delta_{1}(N)\right)^{2}\right]
$$

for any $\delta>\delta_{1}(N)$. Since under $P_{L}^{(N)}$ all $\left|H_{j k}\right| \leq \sqrt{2}(L / \sqrt{N})$, the spectral radius is $\leq 2 L$. Thus, the left hand side of (3.9) is unchanged if we replace $f=f_{z}$ with $f=f_{z}^{L}(t)$, where $f_{z}^{L}(t)=\log (z-t)$ if $|t| \leq 2 L, f_{z}^{L}(t)=\log (z-2 L)$ if $t>2 L$ and $f_{z}^{L}(t)=\log (z+2 L)$ if $t<-2 L$. Now, $f_{z}^{\bar{L}}(t)$ is Lipschitz and there is a constant $C_{3}$, independent of $L$, such that $\left|f_{z}^{L}(t)\right|_{\mathcal{L}} \leq C_{3}$ and $\left\|f_{z}^{L}(t)\right\|_{\mathcal{L}} \leq C_{3}(1+\log L)$ for all $z \in \Omega_{z, \eta}$. Take $L=L_{N}=N^{1 / 2-\xi}$ and $\epsilon=1 / 6$ in (3.8). Then $\delta_{1}(N) \leq C N^{-2 \xi}$ and if we choose $\delta=N^{\xi}$ in (3.9) we obtain

$$
P_{L}^{(N)}\left[\left|\frac{1}{N} \operatorname{Tr} f_{z}(H)-\int_{-1}^{1} f_{z}(t) \sigma(t) d t\right| \geq N^{-\xi}\right] \leq c_{1} \exp \left(-c_{2} N^{2 \xi}\right)
$$

for some positive constants $c_{1}, c_{2}$. If we use $(3.10)$ we see that the probability of the complement of the event in (3.7) can be estimated as

$$
P_{L_{N}}^{(N)}\left[A_{N}\left(N^{-\xi}\right)^{c}\right] \leq C N^{2} e^{-c_{2} N^{2 \xi}}
$$

Set

$$
d \tilde{P}^{(N)}(H)=\left(P_{L_{N}}^{(N)}\left[A_{N}\left(N^{-\xi}\right)\right]\right)^{-1} \chi_{A_{N}\left(N^{-\xi}\right)}(H) d P_{L_{N}}^{(N)} .
$$

Note that $N^{2} / L_{N}^{p}=N^{2-p(1 / 2-\xi)}$, so combining (3.5), (3.7) and (3.11) we obtain the estimate (3.3). From the definition of $A_{N}(\delta)$ we see that (3.4) holds for $z \in D_{N}$, but then a straightforward approximation argument extends it to all $z \in \Omega_{R, \eta}$. This completes the proof of lemma 3.1.

We now come to the central asymptotic result.

Lemma 3.2. Let $\Omega_{R, \eta}$ be as above, let $\xi \in(0,1 / 2]$ and let $K$ be a compact subset of $\mathbb{R}$. Also let $u_{N}$ be a sequence such that $u_{N} \rightarrow u$ as $N \rightarrow \infty$. Furthermore, let $Y_{R, \eta}$ be the set of all $y \in \mathbb{R}^{N}$ such that

$$
\sup _{z \in \Omega_{R, \eta}}\left|\frac{1}{N} \sum_{j=1}^{N} \log \left(z-y_{j}\right)-\int_{-1}^{1} \log (z-t) \sigma(t) d t\right| \leq C N^{-\xi}
$$

for some constant $C$ and all $N \geq 1$, where $\sigma(t)$ is given by (3.1). Then, we can find $R_{0}>0, \eta_{0}>0$ and a constant $C$ such that for all $y \in Y_{R_{0}, \eta_{0}}, \tau \in K$, $|u| \leq \sqrt{1 / 2+2 a^{2}}$ and $N \geq 1$,

$$
\left|\frac{1}{N \rho(u)} \mathcal{K}_{N}\left(u_{N}, u_{N}+\frac{\tau}{N \rho(u)} ; y\right)-\frac{\sin \pi \tau}{\pi \tau}\right| \leq C\left(\left|u-u_{N}\right|+N^{-\xi}\right),
$$

where $\rho(u)$ is given by (1.11). 
Proof. It follows from the formula $(2.20)$ that

$\frac{1}{N \rho(u)} \mathcal{K}_{N}\left(u_{N}, u_{N}+\frac{\tau}{N \rho(u)} ; y\right)=N \int_{\gamma} \frac{d z}{2 \pi i} \int_{\Gamma} \frac{d w}{2 \pi i} h(z, w) g_{N}(z, w) e^{N\left(f_{N}(w)-f_{N}(z)\right)}$,

where $g_{N}(z, w)$ is given by (2.21),

$$
f_{N}(z)=\frac{1}{2 a^{2}}\left(z^{2}-2 u_{N} z\right)+\frac{1}{N} \sum_{j=1}^{N} \log \left(z-y_{j}\right)
$$

and

$$
h(z, w)=\frac{e^{\omega_{0} \tau}}{\tau}\left(e^{-\tau w / a^{2} \rho(u)}-e^{-\tau(w-z) / a^{2} \rho(u)}\right)
$$

We have taken $\omega=\omega_{0} / N \rho(u)$, where $\omega_{0}$ is given by (3.23) below. The integral in (3.14) will be analyzed using a saddle point argument. It follows from (3.12) and Cauchy's integral formula that there is a constant $C$ such that for all $N \geq 1$, $\tau \in K, y \in Y_{R / 2,2 \eta}$ and $|u| \leq \sqrt{1 / 2+2 a^{2}}$,

$$
\begin{aligned}
& \left|f_{N}^{\prime}(z)-f^{\prime}(z)\right| \leq C\left(N^{-\xi}+\left|u-u_{N}\right|\right) \\
& \left|f_{N}^{\prime \prime}(z)-f^{\prime \prime}(z)\right| \leq C N^{-\xi} .
\end{aligned}
$$

A computation shows that,

$$
f^{\prime}(z)=\frac{1}{a^{2}}(z-u)+2\left(z-\sqrt{z^{2}-1}\right) .
$$

Set $S(w)=(w+1 / w) / 2$ with inverse $S^{-1}(z)=z+\sqrt{z^{2}-1}$, where $\sqrt{z^{2}-1}=$ $\sqrt{z-1} \sqrt{z+1}$ (principal argument). The function $S$ maps $\{|w|>1\}$ to $\mathbb{C} \backslash[-1,1]$ and $|w|=1$ is mapped to $[-1,1]$. Note that

$$
f^{\prime}(S(w))=\frac{w}{2 a^{2}}+\left(2+\frac{1}{2 a^{2}}\right) \frac{1}{w}-\frac{u}{a^{2}} .
$$

Write $u=\sqrt{1+4 a^{2}} \cos \theta_{c}$, where $\theta_{c} \in[0, \pi]$. Our assumption on $u$ means that $\left|\cos \theta_{c}\right| \leq 1 / 2$. Note that $f^{\prime}(S(w))=0$ has the solutions $w_{c}^{ \pm}=\sqrt{1+4 a^{2}} \exp \left( \pm i \theta_{c}\right)$. Hence the critical points for $f$ are $z_{c}^{ \pm}=S\left(w_{c}^{ \pm}\right)$.

We will now define some contours that we will use. Pick $\delta>0$ (small), see below. Set, for some $\epsilon>0$ (small), $\gamma_{1}^{+}(t)=S\left(\sqrt{1+4 a^{2}} e^{i \delta}-t\right),-\infty<t \leq 0$, $\gamma_{2}^{+}(t)=S\left(\sqrt{1+4 a^{2}} e^{i t}\right), \delta \leq t \leq \theta_{c}-\epsilon, \gamma_{3}^{+}(t)=S\left(\sqrt{1+4 a^{2}} e^{i t}\right), \theta_{c}-\epsilon \leq t \leq \theta_{c}+\epsilon$, $\gamma_{4}^{+}(t)=S\left(\sqrt{1+4 a^{2}} e^{i t}\right), \theta_{c}+\epsilon \leq t \leq \pi-\delta$ and $\gamma_{5}^{+}(t)=S\left(\sqrt{1+4 a^{2}} e^{i(\pi-\delta)}-t\right)$, $0 \leq t<\infty$. Also, set $\gamma_{j}^{-}(t)=\overline{\gamma_{j}^{+}(t)}, 1 \leq j \leq 5$. Then, we can take $\gamma=$ $\sum_{j=1}^{5}\left(\gamma_{j}^{+}-\gamma_{j}^{-}\right)=\gamma^{+}-\gamma^{-}$in (3.14). Let $t_{0} \in\left(1 / \sqrt{1+4 a^{2}}, 1\right)$ be such that $\operatorname{Im} S\left(t_{0} w_{c}^{+}\right)=\eta$, and write $\alpha=\operatorname{Re} S\left(t_{0} w_{c}^{+}\right)$. Set, for some $\epsilon>0$ (small), $\Gamma_{1}^{+}(t)=$ $\alpha+i t, 0 \leq t \leq \eta, \Gamma_{2}^{+}(t)=S\left(t w_{c}^{+}\right), t_{0} \leq t \leq 1-\epsilon, \Gamma_{3}^{+}(t)=S\left(t w_{c}^{+}\right), 1-\epsilon \leq t \leq 1+\epsilon$ and $\Gamma_{4}^{+}(t)=S\left(t w_{c}^{+}\right), 1+\epsilon \leq t$. Also, set $\Gamma_{j}^{-}(t)=\overline{\Gamma_{j}^{+}(t)}, 1 \leq j \leq 4$. We can then take $\Gamma=\sum_{j=1}^{4}\left(\Gamma_{j}^{+}-\Gamma_{j}^{-}\right)=\Gamma^{+}-\Gamma^{-}$in (3.14). Set

$$
L_{N}^{b d}(\tau ; y)=N \int_{\gamma_{3}^{b}} \frac{d z}{2 \pi i} \int_{\Gamma_{3}^{d}} \frac{d w}{2 \pi i} h(z, w) g_{N}(z, w) e^{N\left(f_{N}(w)-f_{N}(z)\right)},
$$

where $b, d \in\{+,-\}$ and write $L_{N}=L_{N}^{++}-L_{N}^{+-}-L_{N}^{-+}+L_{N}^{--}$. 
Claim 3.3. We can choose $R_{0}>0, \eta_{0}>0$ and $\epsilon, \delta>0$, so that $\gamma_{3}^{+}+\gamma_{3}^{-}+\Gamma_{3}^{+}+\Gamma_{3}^{-}$ lies in a neighbourhood of $z_{c}^{ \pm}$which is included in $\Omega_{R_{0} / 2,2 \eta_{0}}$ and for all $N \geq 1$, $\tau \in K, y \in Y_{r / 2,2 \eta}$ and $|u| \leq \sqrt{1 / 2+2 a^{2}}$,

$$
\left|\frac{1}{N \rho(u)} \mathcal{K}_{N}\left(u_{N}, u_{N}+\frac{\tau}{N \rho(u)} ; y\right)-L_{N}(\tau ; y)\right| \leq C e^{-c N}
$$

with $c>0$

The claim will be proved below. We will now use the claim to finish the proof of lemma 3.2. It follows from (3.15) that there are critical points $z_{N}^{ \pm}=S\left(w_{N}^{ \pm}\right)$for $f_{N}(z)$ such that

$$
\left|z_{N}^{ \pm}-z_{c}^{ \pm}\right| \leq C\left(N^{-\xi}+\left|u-u_{N}\right|\right)
$$

We can deform $\gamma_{3}^{ \pm}\left(\Gamma_{3}^{ \pm}\right)$into contours $\gamma_{N}^{ \pm}\left(\Gamma_{N}^{ \pm}\right)$such that the endpoints are unchanged, $\gamma_{N}^{ \pm}(0)=\Gamma_{N}^{ \pm}(0)=z_{N}^{ \pm}$and $\gamma_{N}^{ \pm}\left(\Gamma_{N}^{ \pm}\right)$have $C^{1}$-distance $\leq C\left(N^{-\xi}+\left|u-u_{N}\right|\right)$ to $\gamma_{3}^{ \pm}\left(\Gamma_{3}^{ \pm}\right)$. We can also asume that these contours are chosen so that $\gamma_{N}^{ \pm}(t)=$ $S\left(w_{N}^{ \pm} e^{ \pm i t}\right)$ and $\Gamma_{N}^{ \pm}(t)=S\left(w_{N}^{ \pm}(1+t)\right)$ for $|t| \ll \epsilon$.

We can now proceed in the standard way with a local saddle point argument in (3.16) and prove that there is a constant $C$ such that

$$
\begin{aligned}
& \left|L_{N}^{b d}(\tau ; y)-h\left(z_{N}^{b}, z_{N}^{d}\right) g_{N}\left(z_{N}^{b}, z_{N}^{d}\right) \frac{2 \pi}{(2 \pi i)^{2}} \frac{\left(\gamma_{N}^{b}\right)^{\prime}(0)\left(\Gamma_{N}^{d}\right)^{\prime}(0) e^{N\left(f_{N}\left(z_{N}^{b}\right)-f_{N}\left(z_{N}^{d}\right)\right)}}{\sqrt{f_{N}^{\prime \prime}\left(z_{N}^{b}\right)\left(\gamma_{N}^{b}\right)^{\prime}(0)^{2}} \sqrt{-f_{N}^{\prime \prime}\left(z_{N}^{d}\right)\left(\Gamma_{N}^{d}\right)^{\prime}(0)^{2}}}\right| \\
& \leq \frac{C}{\sqrt{N}}
\end{aligned}
$$

for all $N \geq 1, \tau \in K, y \in Y_{R_{0}, \eta_{0}}$ and $|u| \leq \sqrt{1 / 2+2 a^{2}}$. Note that $z_{N}^{+}=\overline{z_{N}^{-}}$ and $f_{N}\left(z_{N}^{+}\right)-f_{N}\left(z_{N}^{-}\right)$is purely imaginary. Now, $\left(\gamma_{N}^{b}\right)^{\prime}(0)=b i S^{\prime}\left(w_{N}^{b}\right),\left(\Gamma_{N}^{b}\right)^{\prime}(0)=$ $w_{N}^{b} S^{\prime}\left(w_{N}^{b}\right)$ and a computation shows that

$$
f_{N}^{\prime \prime}\left(z_{N}^{b}\right)\left(\gamma_{N}^{b}\right)^{\prime}(0)^{2}=-f_{N}^{\prime \prime}\left(z_{N}^{b}\right)\left(\Gamma_{N}^{b}\right)^{\prime}(0)^{2}=-f_{N}^{\prime \prime}\left(z_{N}^{b}\right) S^{\prime}\left(w_{N}^{b}\right)^{2}\left(w_{N}^{a}\right)^{2},
$$

which has a positive real part by (3.15) and the fact that $f^{\prime \prime}\left(z_{c}^{b}\right) S^{\prime}\left(w_{c}^{b}\right)^{2}\left(w_{c}^{a}\right)^{2}$ has a positive real part. From (2.21) we see that $g_{N}\left(z_{N}^{b}, z_{N}^{d}\right)=0$ if $b \neq d$ and $g_{N}\left(z_{N}^{b}, z_{N}^{b}\right)=f_{N}^{\prime \prime}\left(z_{N}^{b}\right)$. It follows that

$$
\frac{g_{N}\left(z_{N}^{b}, z_{N}^{b}\right)\left(\gamma_{N}^{b}\right)^{\prime}(0)\left(\Gamma_{N}^{b}\right)^{\prime}(0)}{\sqrt{f_{N}^{\prime \prime}\left(z_{N}^{b}\right)\left(\gamma_{N}^{b}\right)^{\prime}(0)^{2}} \sqrt{-f_{N}^{\prime \prime}\left(z_{N}^{b}\right)\left(\Gamma_{N}^{b}\right)^{\prime}(0)^{2}}}=-b i .
$$

Also, from (3.18) it follows that $\left|h\left(z_{N}^{b}, z_{N}^{b}\right)-h\left(z_{c}^{b}, z_{c}^{b}\right)\right| \leq C\left(N^{-\xi}+\left|u-u_{N}\right|\right)$, and thus 3.19 ) yields

$$
\left|L_{N}^{b d}(\tau ; y)\right| \leq \frac{C}{\sqrt{N}}
$$

if $b \neq d$ and

$$
\left|L_{N}^{b b}(\tau ; y)+\frac{b h\left(z_{c}^{b}, z_{c}^{b}\right)}{2 \pi i}\right| \leq C\left(N^{-\xi}+\left|u-u_{N}\right|\right) .
$$


Combining (3.16), (3.20) and (3.21) we obtain

$$
\left|L_{N}(\tau ; y)+\frac{h\left(z_{c}^{+}, z_{c}^{+}\right)-h\left(z_{c}^{-}, z_{c}^{-}\right)}{2 \pi i}\right| \leq C\left(N^{-\xi}+\left|u-u_{N}\right|\right) .
$$

Now,

$$
h\left(z_{c}^{ \pm}, z_{c}^{ \pm}\right)=\frac{e^{\omega_{0} \tau}}{\tau}\left(e^{-\tau z_{c}^{ \pm} / a^{2} \rho(u)}-1\right)
$$

and a computation shows that

$$
\frac{z_{c}^{ \pm}}{a^{2} \rho(u)}=\pi \frac{1+2 a^{2}}{2 a^{2}} \cot \theta_{c} \pm \pi i \doteq \omega_{0} \pm \pi i
$$

Thus (3.22) becomes

$$
\left|L_{N}(\tau ; y)-\frac{\sin \pi \tau}{\pi \tau}\right| \leq C\left(N^{-\xi}+\left|u-u_{N}\right|\right) .
$$

If we combine this estimate with (3.17) we see that the lemma is proved.

It remains to prove claim 3.3 .

Proof. Let $\gamma_{*}^{ \pm}=\sum_{j \neq 3} \gamma_{j}^{ \pm}$and $\Gamma_{*}^{ \pm}=\sum_{j \neq 3} \Gamma_{j}^{ \pm}$. We have to estimate

$$
I_{1}^{b d}=N \int_{\gamma_{*}^{b}}|d z| \int_{\Gamma^{d}}|d w||h(z, w)|\left|g_{N}(z, w)\right| e^{N \operatorname{Re}\left(f_{N}(w)-f_{N}\left(z_{c}^{d}\right)\right)-N \operatorname{Re}\left(f_{N}(z)-f_{N}\left(z_{c}^{b}\right)\right)}
$$

and

$$
I_{2}^{b d}=N \int_{\gamma^{b}}|d z| \int_{\Gamma_{*}^{d}}|d w||h(z, w)|\left|g_{N}(z, w)\right| e^{N \operatorname{Re}\left(f_{N}(w)-f_{N}\left(z_{c}^{d}\right)\right)-N \operatorname{Re}\left(f_{N}(z)-f_{N}\left(z_{c}^{b}\right)\right)},
$$

where $b, d \in\{+,-\}$. Note that $f_{N}\left(z_{c}^{+}\right)-f_{N}\left(z_{c}-\right)$ is purely imaginary. We will concentrate on $I_{1}^{++}$since the other cases are similar.

Using the inequality

$$
\left|\frac{w-y_{j}}{z-y_{j}}\right|=\left|1+\frac{w-z}{z-y_{j}}\right| \leq 1+C(|w|+|z|)
$$

it is not difficult to see that there are constants $C_{1}$ and $C_{2}$ such that

$|h(z, w)|\left|g_{N}(z, w)\right| e^{N \operatorname{Re}\left(f_{N}(w)-f_{N}(z)\right)} \leq C_{1} E^{C_{2} N(|z|+|w|)+N\left(\operatorname{Re}\left(w^{2}-2 u w\right)-\operatorname{Re}\left(z^{2}-2 u z\right)\right) / 2 a^{2}}$

for all $y \in \mathbb{R}^{N}, \tau \in K$ and $|u| \leq \sqrt{1 / 2+2 a^{2}}$. Note that $|\operatorname{Im} z| \geq c>0$ for all $z \in \gamma$. (The constant $c$ depends on the $\delta$ in the definition of $\gamma$, but as we will see below $\delta$ depends only on the parameter $a$ in the problem.) From the estimate (3.24) it follows that by picking $R=R_{0}$ sufficiently large, the contribution to $I_{1}^{++}$from $z$ and/or $w$ outside $\Omega_{R_{0}, 0}$ is $\leq e^{-N}$. Thus we can assume that $z, w \in \Omega_{R_{0}, 0}$. Next, we will derive the other estimates we will need to prove the claim. 
Assume that $z \in \Omega_{R_{0}, \eta}$ and $w \in \Gamma_{1}^{+}$. Then,

$$
\begin{aligned}
& \left|g_{N}(z, w) e^{N f_{N}(w)}\right| \\
& \leq C\left(1+\frac{1}{N} \sum_{k=1}^{N} \frac{1}{\left|w-y_{k}\right|}\right) \prod_{j=1}^{N}\left|w-y_{j}\right| e^{N \operatorname{Re}\left(w^{2}-2 u w\right) / 2 a^{2}} \\
& \leq C\left(1+\frac{1}{N} \sum_{j=1}^{N} \frac{1}{\left|\alpha+i \eta-y_{j}\right|}\right) \prod_{j=1}^{N}\left|\alpha+i \eta-y_{j}\right| e^{N \operatorname{Re}\left(w^{2}-2 u w\right) / 2 a^{2}} \\
& \leq C e^{N\left[\operatorname{Re} f_{N}(\alpha+i \eta)+\operatorname{Re}\left(w^{2}-2 u w\right)-\left((\alpha+i \eta)^{2}-2 u(\alpha+i \eta)\right)\right] / 2 a^{2}} .
\end{aligned}
$$

If we use (3.12) and the definition of $f_{N}$ we obtain

$$
\begin{aligned}
& \left|g_{N}(z, w) e^{N\left(f_{N}(w)-f_{N}\left(z_{c}^{+}\right)\right)}\right| \\
& \leq C e^{c N\left(N^{-\xi}+\left|u-u_{N}\right|\right)+N \eta^{2} / 2 a^{2}+N \operatorname{Re}\left(f(\alpha+i \eta)-f\left(z_{c}^{+}\right)\right) / 2 a^{2}}
\end{aligned}
$$

for $z \in \Omega_{R_{0}, \eta}$ and $w \in \Gamma_{1}^{+}$.

We will now compute how $\operatorname{Re} f(z)$ changes along $\gamma$. Assume that $\theta_{c} \geq 0$, the other case is analogous. Consider $\gamma(\theta)=S\left(\sqrt{1+4 a^{2}} e^{i \theta}\right), \delta \leq \theta \leq \pi-\delta$. A computation, using the fact that $f^{\prime}\left(\gamma\left(\theta_{c}\right)\right)=0$ gives $\operatorname{Re} \frac{d}{d \theta} f(\gamma(\theta))=\frac{1+2 a^{2}}{2 a^{2}} \sin \theta\left(\cos \theta_{c}-\right.$ $\cos \theta)$. From this we see that there is a constant $c_{0}>0$ such that

$$
\operatorname{Re}\left(f\left(\sqrt{1+4 a^{2}} e^{i \theta}\right)-f\left(z_{c}^{+}\right)\right) \geq c_{0}\left(\theta-\theta_{c}\right)^{2} .
$$

Next, consider $\gamma_{1}(t)=S\left(\sqrt{1+4 a^{2}} e^{i \delta}-t\right), t \leq 0$. If we write $\omega_{\delta}=\sqrt{1+4 a^{2}} e^{i \delta}$, then

$$
\frac{d}{d t} f\left(\gamma_{1}(t)\right)=-\frac{1}{4 a^{2}}\left[\omega_{\delta}-t-2 u+\frac{1+4 a^{2}}{\omega_{\delta}-t}\right]\left[1-\frac{1}{\left(\omega_{\delta}-t\right)^{2}}\right] .
$$

Set $\omega_{\delta}-t=s(t) e^{i \theta(t)}$. A computation shows that

$$
\begin{aligned}
& \operatorname{Re} \frac{d}{d t} f\left(\gamma_{1}(t)\right)=-\frac{1}{4 a^{2} \sqrt{1+4 a^{2}}}\left\{\left[\left(s(t)+\frac{1}{s(t)}\right) \cos \theta(t)-2 \cos \theta_{c}\right]\right. \\
& \left.\times\left[1+4 a^{2}-\frac{1}{s(t)^{2}} \cos 2 \theta(t)\right]-\frac{1}{s(t)^{2}} \sin 2 \theta(t)\left(s(t)-\frac{1}{s(t)}\right) \sin \theta(t)\right\} .
\end{aligned}
$$

Note that $\sin \theta(t)=s(t)^{-1} \sqrt{1+4 a^{2}} \sin \delta$. It follows that the right hand side of (3.27) equals

$$
\begin{aligned}
& -\frac{1}{4 a^{2} \sqrt{1+4 a^{2}}}\left\{\left[\left(s(t)+\frac{1}{s(t)}\right)\left(1+4 a^{2}-\frac{1}{s(t)^{2}}+2 \frac{\left(1+4 a^{2}\right) \sin ^{2} \delta}{s(t)^{4}}\right)\right.\right. \\
& \left.\left.-2 \frac{\left(1+4 a^{2}\right) \sin ^{2} \delta}{s(t)^{4}}\left(s(t)-\frac{1}{s(t)}\right)\right] \cos \theta(t)-2\left(1+4 a^{2}-\frac{1}{s(t)^{2}}+2 \frac{\left(1+4 a^{2}\right) \sin ^{2} \delta}{s(t)^{4}}\right) \cos \theta_{c}\right\}
\end{aligned}
$$

and this is

$$
\leq-\frac{1}{4 a^{2} \sqrt{1+4 a^{2}}}\left(1+4 a^{2}-\frac{1}{s(t)^{2}}\right)\left[\left(s(t)+\frac{1}{s(t)}\right) \cos \theta(t)-2\left(1+\frac{1+4 a^{2}}{2 a^{2}} \sin ^{2} \delta\right) \cos \theta_{c}\right],
$$

since $s(t) \geq 1$. Choose $\delta \leq \theta_{c} / 4$ so that

$$
\left(1+\frac{1+4 a^{2}}{2 a^{2}} \sin ^{2} \delta\right) \cos \theta_{c} \leq \cos \frac{\theta_{c}}{2} .
$$


Since $s(t)+1 / s(t) \geq 2$ and $\theta(t) \leq \delta$ we see that there is a constant $c_{0}>0$ such that

$$
\operatorname{Re} \frac{d}{d t} f\left(\gamma_{1}(t)\right) \leq-c_{0}
$$

For $\gamma_{5}(t)=S\left(\sqrt{1+4 a^{2}} e^{i(\pi-\delta)}-t\right), t \geq 0$, we still have the formula (3.28) with $\gamma_{1}(t)$ replaced by $\gamma_{5}(t)$ and, since $\pi-\delta \leq \theta(t) \leq \pi$, we see that the right hand side is

$$
\geq \frac{1}{\sqrt{1+4 a^{2}}}\left[\left(s(t)+\frac{1}{s(t)}\right) \cos (\pi-\theta(t))+2 \cos \theta_{c}\right]
$$

and consequently there is a constant $c_{0}>0$ such that

$$
\operatorname{Re} \frac{d}{d t} f\left(\gamma_{5}(t)\right) \geq c_{0} \text {. }
$$

Consider now how $\operatorname{Re} f(w)$ changes along $\Gamma^{+}$. Set $\Gamma(t)=S\left(t w_{c}^{+}\right), t \geq t_{0}$. A computation gives

$$
\operatorname{Re} \frac{d}{d t} f\left(S\left(t w_{c}\right)\right)=\frac{1-t}{2 a^{2} t^{2}}\left[1+t\left(1+4 a^{2}\right)-\left(t^{2}\left(1+4 a^{2}\right)+\frac{1}{t}\right) \cos 2 \theta_{c}\right] .
$$

Now, since $|u| \leq \sqrt{1 / 2+2 a^{2}}$, it follows that $\cos 2 \theta_{c} \leq 0$ and thus

$$
\begin{aligned}
& \operatorname{Re} \frac{d}{d t} f\left(S\left(t w_{c}\right)\right) \geq \frac{1-t}{2 a^{2} t^{2}}\left(1+t\left(1+4 a^{2}\right)\right) \quad \text { if } t_{0} \leq t \leq 1 \\
& \operatorname{Re} \frac{d}{d t} f\left(S\left(t w_{c}\right)\right) \leq \frac{1-t}{2 a^{2} t^{2}}\left(1+t\left(1+4 a^{2}\right)\right) \quad \text { if } t \geq 1 .
\end{aligned}
$$

The first of these estimates can be used to show that if we pick $\eta=\eta_{0}$ sufficiently small, then

$$
\eta^{2}+\operatorname{Re}\left(f(\alpha+i \eta)-f\left(z_{c}^{+}\right)\right) \leq-c_{0}
$$

for some positive $c_{0}$. If we use this in (3.25) we obtain

$$
\left|g_{N}(z, w) e^{N\left(f_{N}(w)-f_{N}\left(z_{c}^{+}\right)\right)}\right| \leq C e^{-c_{0}^{\prime} N}
$$

for some positive $c_{0}^{\prime}$. We can now use (3.26), (3.29), (3.31), (3.32) and (3.33) to estimate $I_{1}^{++}$and see that it is $\leq C e^{-c N}$ for some positive $c$.

\section{Proof of the TheOREMS}

We start with the proof of theorem 1.2 .

Proof. By proposition 1.1 and Fubini's theorem the integral in the left hand side of (1.14) can be written

$$
\int_{\mathcal{H}_{N}}\left(\int_{\mathbb{R}^{N}} \rho_{N}(x, y(H))(S f)\left(N \rho(u)\left(x_{1}-u\right), \ldots, N \rho(u)\left(x_{N}-u\right)\right) d^{N} x\right) d P^{(N)}(H)
$$

Note that $\|S(f)\|_{\infty} \leq N^{m}\|f\|_{\infty}$. Since $\rho_{N}(x, \cdot)$ is a probability density on $\mathbb{R}^{N}$ we can use lemma 3.1 to replace the expression in (4.1) by

$$
\int_{\mathcal{H}_{N}}\left(\int_{\mathbb{R}^{N}} \rho_{N}(x, y(H))(S f)\left(N \rho(u)\left(x_{1}-u\right), \ldots, N \rho(u)\left(x_{N}-u\right)\right) d^{N} x\right) d \tilde{P}^{(N)}(H)
$$


with an error $\leq C N^{m}\|f\|_{\infty} N^{2-p(1 / 2-\xi)}=o(1)$, since $p>2(m+2)$, provided we choose $\xi$ small enough. Now, since $\rho_{N}(x, \cdot)$ is symmetric it follows from (1.13), (2.4), (2.14) and (2.19) that the expression in (4.2) can be written

$$
\begin{array}{rl}
\int_{\mathcal{H}_{N}} \int_{\mathbb{R}^{m}} & f\left(t_{1}, \ldots, t_{m}\right) \\
& \times \operatorname{det}\left(\frac{1}{N \rho(u)} \mathcal{K}\left(u+\frac{t_{i}}{N \rho(u)}, u+\frac{t_{j}}{N \rho(u)} ; y(H)\right)\right)_{i, j=1}^{m} d^{m} t d \tilde{P}^{(N)}(H) .
\end{array}
$$

Since $f$ has compact support and we know that (3.4) holds a.s. $\left[\tilde{P}^{(N)}\right]$ it follows from lemma 3.2, with $u_{N}=u+t_{i} / N \rho(u), \tau=t_{j}-t_{i}$, that

$$
\left|\mathcal{K}\left(u+\frac{t_{i}}{N \rho(u)}, u+\frac{t_{j}}{N \rho(u)} ; y(H)\right)-\frac{\sin \pi\left(t_{i}-t_{j}\right)}{\pi\left(t_{i}-t_{j}\right)}\right| \leq C N^{-\xi},
$$

for a.a. $\left[\tilde{P}^{(N)}\right]$ and all $\left(t_{1}, \ldots, t_{m}\right)$ in the support of $f$. Thus we can take the limit as $N \rightarrow \infty$ in (4.3) and obtain the right hand side of (1.14). This completes the proof.

Before proving theorem 1.3 we need some preliminary results on the level spacing distribution. Let $\rho_{N}(x)$ be a symmetric probability density on $\mathbb{R}^{N}$ with correlation functions defined by (1.1). Assume that $R_{1}^{(N)} / N \rightarrow \rho(t)$ (weakly) as $N \rightarrow \infty$, so that $\rho(t)$ is the asymptotic density. Let $u$ be a given point such that $\rho(u)>0$, and let $t_{N}$ be a sequence such that $t_{N} \rightarrow \infty$ but $t_{N} / N \rightarrow 0$ as $N \rightarrow \infty$. Set, for $|r| \leq 1 / 2$,

$$
\mathcal{R}_{m}^{(N)}\left(\sigma_{1}, \ldots, \sigma_{m} ; r\right)=\frac{1}{(N \rho(u))^{m}} R_{m}^{(N)}\left(u+\frac{2 t_{N} r+\sigma_{1}}{N \rho(u)}, u+\frac{2 t_{N} r+\sigma_{m}}{N \rho(u)}\right)
$$

and let $\mathcal{R}_{m}\left(\sigma_{1}, \ldots, \sigma_{m}\right)$ be the limiting correlation functions, which we assume are continuous, symmetric and translation invariant. Assume that, for each $s \geq 0$,

$$
D_{N}(s)=\sum_{m=N+1}^{\infty} \frac{s^{m}}{m !} \sup _{\left|\sigma_{j}\right| \leq s}\left|\mathcal{R}_{m}\left(\sigma_{1}, \ldots, \sigma_{m}\right)\right|<\infty .
$$

Set

$$
H(s)=\sum_{m=0}^{\infty} \frac{(-1)^{m}}{m !} \int_{[0, s]^{m}} \mathcal{R}_{m}\left(\sigma_{1}, \ldots, \sigma_{m}\right) d^{m} \sigma
$$

(the probability of no particle in $[0, s]$ ), which is well defined by (4.4). Also, set

$$
\epsilon_{m}^{(N)}=\sup _{\left|\sigma_{j}\right| \leq s,|r| \leq 1 / 2}\left|\mathcal{R}_{m}^{(N)}\left(\sigma_{1}, \ldots, \sigma_{m} ; r\right)-\mathcal{R}_{m}\left(\sigma_{1}, \ldots, \sigma_{m}\right)\right| .
$$

Proposition 4.1. Let $S_{N}(s, x)$ be defined by 1 1.5). Then

$$
\left|\int_{\mathbb{R}^{N}} S_{N}(s, x) \rho_{N}(x) d^{N} x-\int_{0}^{s} H^{\prime \prime}(u) d u\right| \leq D_{N}(s)+\sum_{m=2}^{N} \frac{s^{m-1}}{(m-1) !} \epsilon_{m}^{(N)} .
$$

Proof. We first show that

$$
\int_{0}^{s} H^{\prime \prime}(u) d u=\sum_{m=2}^{N} \frac{s^{m-1}}{(m-1) !} \int_{[0, s]^{m-1}} \mathcal{R}_{m}\left(0, \tau_{2}, \ldots, \tau_{m}\right) d \tau_{2} \ldots d \tau_{m},
$$


see [7. Since $\mathcal{R}_{m}$ is translation invariant and symmetric by assumption, we have

$$
\begin{aligned}
H^{\prime}(u) & =\lim _{\epsilon \rightarrow 0} \frac{1}{\epsilon} \sum_{m=0}^{\infty} \frac{(-1)^{m}}{m !} \int_{[-\epsilon, u]^{m} \backslash[0, u]^{m}} \mathcal{R}_{m}\left(x_{1}, \ldots, x_{m}\right) d^{m} x \\
& =\lim _{\epsilon \rightarrow 0} \sum_{m=0}^{\infty} \frac{(-1)^{m}}{m !} \frac{1}{\epsilon}\left(m \int_{[-\epsilon, 0] \times[0, u]^{m-1}} \mathcal{R}_{m}\left(x_{1}, \ldots, x_{m}\right) d^{m} x\right) \\
& =\sum_{m=1}^{\infty} \frac{(-1)^{m}}{(m-1) !} \int_{[0, u]^{m-1}} \mathcal{R}_{m}\left(0, x_{2}, \ldots, x_{m}\right) d^{m-1} x,
\end{aligned}
$$

where we have also used (4.4) and the continuity of $\mathcal{R}_{m}$. Continuing in the same way we see that $H(u)$ is actually a $C^{\infty}$ function, in particular $H^{\prime \prime}(u)$ is well defined and continuous. From (4.8) we get

$$
H^{\prime}(s)=-\mathcal{R}_{m}(0)+\sum_{m=2}^{\infty} \frac{(-1)^{m}}{(m-1) !} \int_{[0, s]^{m-1}} \mathcal{R}_{m}\left(0, x_{2}, \ldots, x_{m}\right) d^{m-1} x .
$$

Hence $H^{\prime}(0)=-\mathcal{R}_{m}(0)$ and we see that the right hand side of (4.7) equals $H^{\prime}(u)-$ $H^{\prime}(0)$, which is what we wanted to prove.

It is proved in [7], using a result from [17], that

$$
\begin{aligned}
& \int_{\mathbb{R}^{N}} S_{N}(s, x) \rho_{N}(x) d^{N} x \\
& =\sum_{m=2}^{N} \frac{(-1)^{m}}{(m-1) !} \int_{-1 / 2}^{1 / 2} d r \int_{\left[0, \min \left(s,(1-2 r) t_{N}\right)\right]^{m-1}} \mathcal{R}_{m}^{(N)}\left(0, \sigma_{2}, \ldots, \sigma_{m} ; r\right) d^{m-1} \sigma .
\end{aligned}
$$

Hence, the estimate (4.6) follows from (4.4), (4.5) and (4.7).

We turn now to the proof of theorem 1.3 .

Proof. Just as in the proof of theorem 1.2 above we see that since $P \in \mathcal{W}^{6+\epsilon}$ and $\left\|S_{N}\right\|_{\infty} \leq N / 2 t_{N}$

$$
\begin{aligned}
& \left|\int_{\mathcal{H}_{N}} S_{N}(s, x(M)) d Q^{(N)}(M)-\int_{\mathcal{H}_{N}}\left(\int_{\mathbb{R}^{N}} S_{N}(s, x) \rho_{N}(x ; y(H)) d^{N} x\right) d \tilde{P}^{(N)}(H)\right| \\
& \leq C \frac{N}{t_{N}} N^{2-(6+\epsilon)(1 / 2-\xi)} \leq \frac{C}{t_{N}},
\end{aligned}
$$

if we take $\xi$ sufficiently small, and also that (3.4) holds. From proposition 1.1, (2.4) and proposition 2.3 we know the correlation functions of $\rho_{N}(x ; y)$, and if we take $u_{N}=u+\left(2 t_{N} r+\sigma_{i}\right)(N \rho(u))^{-1}$ in lemma 3.2 we see that

$$
\begin{aligned}
\left|\frac{1}{N \rho(u)} \mathcal{K}\left(u+\frac{2 t_{N} r+\sigma_{i}}{N \rho(u)}, u+\frac{2 t_{N} r+\sigma_{j}}{N \rho(u)} ; y(H)\right)-\frac{\sin \pi\left(\sigma_{i}-\sigma_{j}\right)}{\pi\left(\sigma_{i}-\sigma_{j}\right)}\right| & \leq C\left(\frac{t_{N}}{N}+N^{-\xi}\right) \\
& \doteq \omega_{N}
\end{aligned}
$$

for a.a. $H\left[\tilde{P}^{(N)}\right]$. Thus, the limiting correlation functions are

$$
\mathcal{R}_{m}\left(\sigma_{1}, \ldots, \sigma_{m}\right)-\operatorname{det}\left(\frac{\sin \pi\left(\sigma_{i}-\sigma_{j}\right)}{\pi\left(\sigma_{i}-\sigma_{j}\right)}\right)_{i, j=1}^{m} .
$$


Since the matrix in the determinant is positive definite it follows from the Hadamard inequality that

$$
D_{N}(s) \leq \sum_{m=N+1}^{\infty} \frac{s^{m}}{m !}
$$

Also, since

$$
\mathcal{R}_{m}^{(N)}\left(\sigma_{1}, \ldots, \sigma_{m} ; y\right)=\operatorname{det}\left(\frac{1}{N \rho(u)} \mathcal{K}\left(u+\frac{2 t_{N} r+\sigma_{i}}{N \rho(u)}, u+\frac{2 t_{N} r+\sigma_{j}}{N \rho(u)} ; y\right)\right)_{i, j=1}^{m}
$$

it follows from (4.10), the multilinearity of the determinant and Hadamard's inequality that

$$
\left|\mathcal{R}_{m}^{(N)}(\sigma ; y)-\mathcal{R}_{m}(\sigma)\right| \leq m\left(1+\omega_{N}\right)^{m-1} \omega_{N} m^{m / 2},
$$

and hence $\epsilon_{m}^{(N)} \leq m\left(1+\omega_{N}\right)^{m-1} \omega_{N} m^{m / 2}$. Now, by proposition 4.1, Stirling's formula and the fact that $\omega_{N} \rightarrow 0$,

$$
\begin{aligned}
& \left|\int_{\mathbb{R}^{N}} S_{N}(s, x) \rho_{N}(x ; y(H)) d^{N} x-\int_{0}^{s} H^{\prime \prime}(u) d u\right| \\
& \leq \sum_{m=N+1}^{\infty} \frac{s^{m}}{m !}+\omega_{N} \sum_{m=2}^{N} \frac{s^{m}}{(m-1) !}\left(1+\omega_{N}\right)^{m-1} m^{(m+2) / 2}=o(1)
\end{aligned}
$$

as $N \rightarrow \infty$, for a.a. $H\left[\tilde{P}^{(N)}\right]$. If we combine 4.9 and 4.11 we see that the theorem is proved.

\section{REFERENCES}

[1] C. Andréief, Note sur une relation les integrales définies des produits de fonctions, Mém. de la Soc. Bordeaux, 2 (1883), 1 - 14

[2] Z. D. Bai, Methodologies in spectral analysis of large dimensional random matrices: a review, Statistica Sinica, 9 (1999), 611 - 661

[3] P. Bleher, A. Its, Semiclassical asymptotics of orthogonal polynomials, Riemann-Hilbert problem, and universality in the matrix model, Ann. Math., 150 (1999), 185 - 266

[4] E. Brézin, S. Hikami, Correlations of nearby levels induced by a random potential, Nucl. Phys. B, 479, (1996), 697 - 706

[5] E. Brézin, S. Hikami, Spectral form factor in a random matrix theory, Phys. Rev. E, 55, (1997), 4067 - 4083

[6] E. Brézin, S. Hikami, S., An extension of level-spacing universality, cond-mat/9702213

[7] P. Deift, T. Kriecherbauer, K. T-R McLaughlin, S. Venakides, X. Zhou, Uniform asymptotics for polynomials orthogonal with respect to varying exponential weights and applications to universality questions in random matrix theory, Comm. Pure. Appl. Math., 52 (1999), 1335 $-1425$

[8] F. J. Dyson, A Brownian-motion Model for the Eigenvalues of a Random Matrix, J. Math. Phys., 3 (1962), 1191 - 1198

[9] D. J. Grabiner, Brownian motion in a Weyl chamber, non-colliding particles and random matrices, Ann. Inst. H. Poincaré, 35 (1999), 177 - 204

[10] A. Guionnet, O. Zeitouni, Concentration of the spectral measure for large matrices, preprint (2000)

[11] Harish-Chandra, Differential operators on a semisimple Lie algebra, Am J. Math., 79, (1957), $87-120$

[12] D. G. Hobson, W. Werner, Non-colliding Brownian motions on the circle, Bull. London Math. Soc., 28 (1996), 543 - 650 
[13] C. Itzykson, J. -B. Zuber, The planar approximation II, J. Math. Phys., 21, (1980), 411 421

[14] K. Johansson, Random growth and Random matrices, to appear in the Proceedings of the third European Congress of Mathematics

[15] K. Johansson, Non-intersecting paths, random tilings and random matrices, in preparation

[16] S. Karlin, G. McGregor, Coincidence probabilities, Pacific J. Math, 9 (1959), 1141 - 1164

[17] N. M. Katz, P. Sarnak, Random Matrices, Frobenius Eigenvalues and Monodromy, AMS Colloquium Publications, Vol. 45, 1999

[18] A. Khorunzhy, On smoothed density of states for Wigner random matrices, Random Oper. and Stoch. Equ., 5, (1997), 147 - 162

[19] A. Khorunzhy, B. A. Khoruzhenko, L. A. Pastur, On asymptotic properties of large rando matrices with independent entries, J. Math. Phys., 37, (1996), 5033 - 5060

[20] M. L. Mehta, Random Matrices, 2nd ed., Academic Press, San Diego 1991

[21] A. Okounkov, Infinite wedge and measures on partitions, math.RT/9907127

[22] L. A. Pastur, M. Shcherbina, Universality of the local eigenvalue statistics for a class of unitary invariant random matrix ensembles, J. Stat. Phys., 86, (1997), 109 - 147

[23] E. J. Pauwels, L. G. G. Rogers, Skew-product decompositions of Brownian motions in Geometry of Random Motion, R. Durrett, M. A. Pinsky, eds., AMS Contemporary Mathematics, Vol. 73,1988

[24] R. G. Pinsky, On the convergence of diffusion processes conditioned to remain in a bounded region for large time to limiting positive recurrent diffusion processes, Ann. Prob., 13 (1985), $363-378$

[25] C. E. Porter, ed., Statistical Theories of spectra: Fluctuations, Academic Press, New York, 1965

[26] A. Soshnikov, Universality at the edge of the spectrum in Wigner random matrices, Commun. Math. Phys., 207 (1999), 697 - 733

[27] Ya. Sinai, A. Soshnikov, Central limit theorem for traces of large random symmetric matrices with independent matrix elements, Bol. Soc. Brasil. Mat., 29 (1998), 1 - 24

[28] Ya. Sinai, A. Soshnikov, A refinment of Wigner's semicircle law in a neighborhood of the spectrum edge for symmetric matrices, Funct. Anal. Appnl., 32 (1998), 114 - 131

[29] C. A. Tracy, H. Widom, Correlation Functions, Cluster Functions, and Spacing Distributions for Random Matrices, J. Statist. Phys., 92, (1998), 809 - 835

Department of Mathematics, Royal Institute of Technology, S-100 44 Stockholm, SWEDEN

E-mail address: kurtj@math.kth.se 